\title{
LEARNING GROUP DIFFERENCES: IMPLICATIONS FOR CONTRAST AND ASSIMILATION IN STEREOTYPING
}

Susanne K. Hicklin and Douglas H. Wedell

University of South Carolina

Three studies examined how the learning environment during stereotype acquisition influences judgments of group members. In Experiments 1 and 2 , participants learned to identify group members by schematic facial features, while supplemental behavioral information was presented incidentally. Experiment 3 reversed the learning and supplemental dimensions. The salience and correlational structure of dimensions was also manipulated across experiments. When information was well individuated, contrast effects tended to occur for dominance judgments of remembered facial feature widths and likableness of behaviors for group members; otherwise assimilation effects or illusory correlation effects tended to occur. Assimilation of ideal points toward group norms was found for judgments of remembered pleasantness of faces, except when faces were learned supplementally. These results demonstrate that how stereotypes are learned can affect the individuation of group member information, which in turn can affect whether stereotypes are used in an assimilative or contrastive fashion in judgment.

Walter Lippman (1922) defined stereotypes as "the little pictures we carry around inside our heads." Decades later, a similar, but less ambiguous definition suggests that stereotypes are the beliefs about a group's personal attributes (Ashmore \& Del Boca, 1981). Now an extensive body of stereotype literature has investigated how and when stereotypes are activated and the implications of stereotype activation for judgments of group members

\footnotetext{
Pleass address correspondence to Susanne K. Hicklin, Department of Psychology, University of South Carolina, Columbia, SC 29208; susannehicklin@hotmail.com
} 
(Blair, 2002; Fiske, 1998; Hamilton \& Sherman, 1994; Hilton \& von Hippel, 1996). Research on preexisting stereotypes, such as race, ethnicity, and gender, has demonstrated that stereotypes for these social groups are prevalent and potent (Deaux, 1985; Lott \& Saxon, 2002; Wittenbrink, Gist, \& Hilton, 1997).

Although the ecological validity of studying preexisting stereotypes is high, there are at least two problems associated with these studies. First, researchers cannot control the content of the stereotype. For instance, Jackson and colleagues (1996) have shown that stereotypes of Asians varied greatly. As Alexander, Brewer, and Livingston (2005) have suggested, there is a recent heightened interest in the actual content of specific stereotypes, and several competing theories of stereotype content have been developed (Eagly, Wood, \& Diekman, 2000; Fiske, Cuddy, Glick, \& Xu, 2002; Johannesen-Schmidt \& Eagly, 2002). Second, in these studies it is difficult to examine the experiences that have led to the acquisition of a specific stereotype. One individual may directly experience behaviors linked to a specific stereotype, while another may only indirectly experience cultural portrayals of the stereotype. It is not clear how these experiences alter stereotype construction or use.

An alternative approach to studying stereotypes is to control the nature of the stereotype and the way that it is acquired by creating groups for individuals to learn.

Using unfamiliar stimuli eliminates confounds that are associated with established stereotypes and enables stereotypes to be systematically and effectively manipulated. Only a limited number of studies have incorporated such stereotype learning paradigms (Biernat \& Crandall, 1996; Manis, Nelson, \& Shedler, 1988; Manis, Paskewitz, \& Cotler, 1986; Pettibone, 2000; Pettibone \& Wedell, in press). Although novel stereotypes generated from these studies have limited generalizability and are possibly low in mundane realism, it seems likely that the psychological processes involved are similar to those used with real-world stereotypes. Studies of learned stereotypes in conjunction with those of existing stereotypes combine to paint a more comprehensive picture of judgments based on stereotypes.

The research by Manis et al. (1986) will be discussed in some detail as the current research builds on this type of learning situa- 
tion. Manis et al. constructed stereotypes based on type of hospital (general or psychiatric) and other correlated cues (handwriting style and patient-generated definitions). In several experiments they attributed definitions varying in the level of implied psychopathology to patients from one of two hospitals. Specifically, patients from Central Hospital typically provided definitions implying high psychopathology, whereas patients from Metropolitan Hospital provided definitions implying low psychopathology. Furthermore, Central Hospital was characterized either as a general or a psychiatric hospital. During an induction phase, a definition and hospital name were presented for participants to view as they decided whether the specific patient from the given hospital was schizophrenic. Later when presented with definitions of midscale psychopathology attributed to patients from each hospital, participants indicated which patient appeared to be more disturbed and gave confidence ratings for the choice. Results demonstrated that stereotype usage depended on features of the induction phase. Before further describing how stereotypes are applied in judgments, it is essential to acknowledge two distinct types of judgment and discuss how they relate to group effects resulting from the learned stereotypic information.

\section{TWO TYPES OF JUDGMENT}

An important feature of our studies is the distinction between dominance and ideal-point judgments described by Wedell and Pettibone (1999) and based on Coombs (1964). In dominance judgment domains, ratings of stimuli consistently increase (or decrease) as stimulus values increase (or decrease)—-that is, judgments are monotonically related to stimulus values. For example, in studies that we will describe, participants rated the perceived widths of facial features related to eyes and nose. These ratings are dominance-based because an increase in feature width leads to an increase in rated value. Many descriptive ratings are dominance-based, whether describing perceived or inferred attributes. For example, ratings of perceived kindness would increase as the number of kind acts of an individual increases, a dominance relationship. Dominance relationships can apply to affective do- 
mains as well, such as when ratings of happiness associated with winnings increase as the amount of winnings increases. In our research, we examined the affective-based dominance domain of likableness of individuals based on behavioral descriptions: The more positive the behaviors described, the more the individual should be liked.

Unlike these types of dominance domains, ideal-point domains may be characterized by single-peaked functions, with ratings of stimuli increasing to a point (i.e., the ideal) and then decreasing with further increases in magnitude of an underlying attribute. The ideal point itself can be conceived as one's attitudinal position on the continuum. For example, without any decorations or paintings adorning the walls, a room may appear barren, sterile, and dull. With too many decorations, a room may look gaudy and distasteful. One's attitude toward the appropriate amount of decorations presumably resides between these two extremes, reflected in an ideal point or attitudinal position. This same relationship is found in more traditional attitude domains. For example, if an individual is moderately liberal, then that person would dislike positions to the degree that they were more liberal or more conservative. The distinction between the dominance and ideal-point domains becomes especially important when context effects are considered, as will be discussed in the next section.

In the research that we report, pleasantness ratings of facial configurations were conceived as ideal-point judgments. This is because participants' perceptions of the pleasantness of faces increased to a point with increase in the feature width, but then perceptions of pleasantness decreased with continued increase of the feature width. In other words, participants thought that some noses (or eye gaps) were too wide and that some were too narrow, with the ideal lying at a moderate value, which we can designate as the ideal or the participant's attitudinal position. The judgment process differs somewhat for dominance and ideal-point judgments. For dominance judgments, one need only determine how much of an attribute a given stimulus has. For ideal-point judgments, one must also compare this attribute value to a preferred attribute value, the ideal. The specification of the ideal is a major source of individual differences and leads to participants having 
different preference orderings for the targets. Indeed, Coombs (1964) developed statistical unfolding models for determining underlying attribute scales from differences in preference orderings.

Most research on stereotypes can be characterized as using dominance judgments as the key dependent variable. Research focusing on ideal-point judgments has been underrepresented in this literature. Indeed most of the studies exploring ideal-point judgments outside of traditional attitude research have centered on less socially relevant stimuli (Holbrook \& Anand, 1990; Riskey, Parducci, \& Beauchamp, 1979), with only a few studies relating to social groups or socially relevant judgments (Pettibone, 2000; Pettibone \& Wedell, in press; Wedell \& Pettibone, 1999; Wedell, Santoyo, \& Pettibone, 2005). The current studies continue this latter line of research.

\section{ASSIMILATION AND CONTRAST FOR DOMINANCE JUDGMENTS}

Most context effects for dominance judgments may be described as either assimilation or contrast effects (for a review see Wedell, Hicklin, \& Smarandescu, 2007). Context effects for dominance judgments shift the response curve up or down, but the ordering of the stimuli is preserved. For example, a typical finding in the stereotype literature is that judgments of an individual tend to be displaced toward values represented in the group stereotype, an assimilation effect. More generally, assimilation occurs when judgments are displaced toward the values of the contextual set of stimuli, as depicted in the downward shift in the rating function in the left panel of Figure 1. For example, when a construction worker and a housewife are described as engaging in the same generally ambiguous aggressive act, such as when the individual "hit someone who annoyed him or her," the construction worker is rated as more aggressive than the housewife (Kunda \& Sherman-Williams, 1993). In this case, the contextual information represented by the stereotype is used to disambiguate the behavior and bring it in line with implications of the stereotype. Assimilation effects are also observed when judgments are made in the absence of individuating information and when individuating in- 


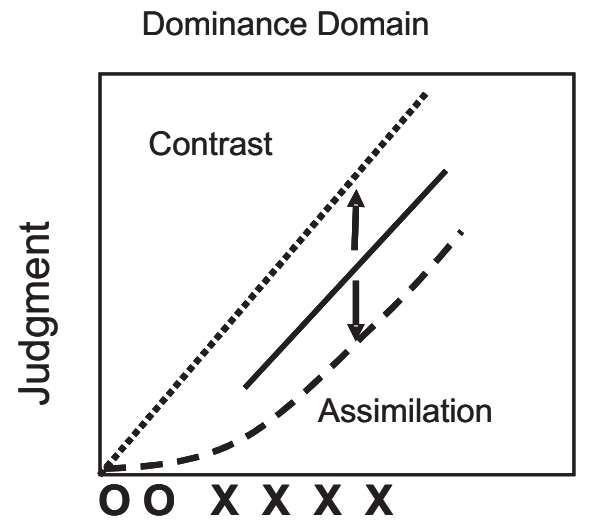

Ideal-Point Domain

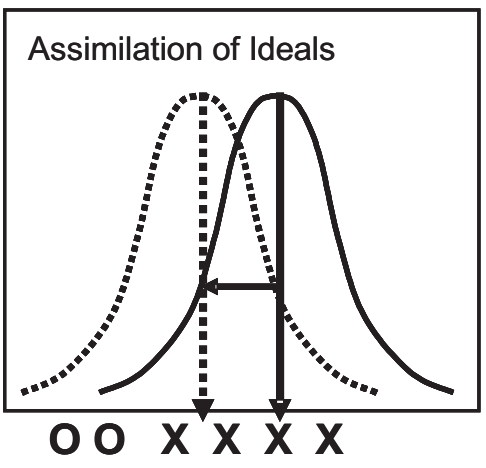

Attribute Value

FIGURE 1. The left panel depicts contrast and assimilation effects for dominance domains and the right panel depicts assimilation for ideal-point domains. The Os represent contextual values and Xs represent target stimuli. The solid line represents judgments of target stimuli in isolation. The dashed lines represent a shift in judgment when contextual stimuli are included in the set.

formation is available but not attended to during encoding (Brewer, 1988; Brewer \& Feinstein, 1999; Fiske \& Pavelchak, 1986). Indeed, the literature emphasizes that assimilation is the typical way that stereotypes are used in social judgment (Hilton \& von Hippel, 1996).

Often when assimilation occurs, the stereotype operates as an interpretative frame for subsequent judgments. However, stereotypes may be used in a different way-that is, activated information gleaned from stereotypes may operate as a comparison standard against which targets are evaluated (Biernat \& Manis, 1994; Biernat, Kobrynowicz, \& Weber, 2003; Biernat, Manis, \& Nelson, 1991; Stapel \& Koomen, 1998). In their studies, Manis et al. (1986) hypothesized that participants implicitly compared the target definition to other definitions from the same hospital, using stereotypes as standards of comparison to produce contrast effects. Thus, ratings of the target were shifted away from stereotypic values, as illustrated by the upward shift of the rating function in the left panel of Figure 1. Such contrast effects can be found with preexisting stereotypes. For example, Kwong See and Heller 
(2004) studied the stereotype that very old individuals have poor language performance compared to younger individuals. Their participants rated transcribed descriptions attributed to young and old individuals for discourse quality. When presented with a good-quality description attributed to a very old individual, participants rated the description as exceptional in discourse quality, whereas the same description was rated as moderate in discourse quality when the description was attributed to a young individual, an example of contrast away from the stereotypic values.

Manis et al. (1986) explored the effects of learning context by varying the location of a stimulus along a dimension of psychopathology. Stereotypes were formed in the induction phase as participants learned correlated cues: Levels of psychopathology for definitions given by patients from one of two hospitals. When Central Hospital was comprised of more disturbed patients than Metropolitan Hospital (learned through a case-by-case presentation), contrast effects were observed so that Central Hospital patients were rated as less disturbed than patients from Metropolitan Hospital. In an alternate condition, the cues were uncorrelated in the induction phase and Central Hospital was depicted as a psychiatric hospital (and Metropolitan Hospital as a general hospital). Assimilation effects were observed in this uncorrelated condition, such that patients from Central Hospital were rated as more disturbed than patients from Metropolitan Hospital. Hence, facets of the induction phase may be critical determinants of how stereotypes are used in judgment.

The primary intent of the current set of studies was to develop a broader understanding of how learning impacts stereotyping in social judgment for dominance and ideal-point domains. Although past research has extensively examined the impact of categorical information on context effects, the learning paradigm that we use in the current studies offers a new way to examine this relationship. Generally, stereotypes are formed over a very long period of time and established stereotypes are often applied to new members of the group. Our interest focuses on the early stages of stereotype formation and application. During this early phase of stereotype generation, stereotypes may be applied to the 
group members who were used to construct the stereotypes. For example, suppose that before attending college one does not have a well-formed stereotype of college professors. During the first semester, a student may interact with five professors, and through these experiences develop the unfortunate stereotype that college professors are arrogant. When asked later by a friend to evaluate one of the five professors, the student will have available in memory individuating information relating to the specific behaviors of that professor along with categorical stereotype information based on the broader set of group members. Our research asks the question of how the stereotype information may affect evaluations of the specific group member. If the stereotype is being used as an interpretative frame during an inference process, the professor may be judged as more arrogant than his or her behaviors warrant, an assimilation effect reflecting the use of group information to disambiguate the individuating information. However, if the student has a clear memory of the behaviors associated with this professor, the stereotype may be used as a standard of comparison so that the professor is judged as less arrogant than his or her behaviors warrant, a contrast effect (Biernat \& Manis, 1994; Biernat et al., 2003; Biernat et al., 1991; Stapel \& Koomen, 1998).

\section{ASSIMILATION AND CONTRAST FOR IDEAL-POINT JUDGMENTS}

As with dominance judgments, context effects on ideal-point judgments may be described as either assimilative or contrastive. Unlike dominance judgments, contextual values promote shifts of the actual ideal point, rather than shifts in the ratings (as is the case in dominance judgments). It is important to note that by folding the scale around the ideal point, the contextual shifts along the folded dimension may be very similar to assimilation and contrast for dominance judgments, essentially shifting judgments up or down. However, shifts in the ideal around which the scale may be folded result in a qualitatively different type of effect. Thus, a critical difference between ideal-point and dominance domains is that individual differences and contextual effects are repre- 
sented very differently. In dominance domains, these are primarily represented in terms of differences in slopes and intercepts, preserving stimulus ordering on the response scale. However, in the ideal-point domain, these may also be represented in shifts in the location of the response function's peak, resulting in different orderings of the stimuli. In affective domains, different orderings of the stimuli tend to indicate reversals of preference. A demonstration of preference reversals is interesting as it reveals a qualitative difference in the valuation process so that one person may be preferred to another in one context, but that preference ordering may be reversed in an alternate context.

Ideal-point judgments are largely understudied, and when examined they tend to reflect assimilative shifts of ideals. As shown in the right panel of Figure 1, assimilation of ideals occurs when the ideal point is shifted horizontally toward the contextual or stereotypic values. Contrast of ideals may be described as occurring when the ideal point is displaced away from contextual values. Contrast of ideal-point judgments has been noted rarely in the literature (for exceptions see Cooke, Janiszewski, Cunha, Nasco, \& de Wilde, 2004).

Wedell et al. (2005) explored socially relevant ideal-point judgments of preference for body images in a series of experiments in which participants judged the pleasantness of human figures varying in body width. They demonstrated assimilation of ideals so that the ideal body width was narrower after viewing silhouettes or computer-generated body images that were predominantly narrow. In the narrow context, body image \#5 tended to be the ideal and in the wide context body image \#11 tended to be the ideal (with numbers corresponding directly to how wide the image was). This shift in ideals resulted in large reversals of preference so that in the narrow context, body image \#5 was rated as much more attractive than body image \#11, but the reverse was true in the wide context. These qualitative differences in preference reflect a systematic shift of attitudes toward thinness of body images and not just quantitative shifts in magnitude of responding. Assimilative shifts of the ideal point were observed for all participants except female participants who were dissatisfied with their own bodies: These individuals maintained an 
unrealistically "thin ideal" regardless of the distribution of body widths to which they were exposed.

Our research examines the question of whether the stereotyping process includes the formation of different ideals associated with different groups so that group members are evaluated relative to group ideals. If this is the case, then we should find that the same set of facial features leads to different pleasantness evaluations depending on the group membership of the target. Although people generally like to appear fair and consistent in their judgments and behaviors, such a finding would reflect a clear double standard being applied across groups, so that different attitudes are applied to the same ostensive features. Our experimental paradigm will allow us to examine how robust this relationship is across different learning environments.

\section{THE INDIVIDUATION HYPOTHESIS}

Past research has examined context effects using well-established stereotypes. The general finding is that individuals use stereotypes as a source of information when they have little knowledge about a specific group member, thereby resulting in assimilation effects. Our research complements these studies by investigating whether these same effects occur when categorical information is initially being acquired and when individual impressions are first developed. Within our learning paradigm we will examine the role of individuating information. Specifically, we ask whether people will ignore learned stereotypes and make bias-free judgments when individuating information is present.

1. Note that the concept of individuation may be related to the concept of distinctiveness, which is often used to predict contrast and assimilation (Stapel, Koomen, \& Velthuijsen, 1998; Stapel \& Winkielman, 1998). We prefer to use the term "individuation" for several reasons. First, one application of the distinctiveness concept has been to distinguish exemplars versus traits, with the latter being of low distinctiveness. However, recent research demonstrates that this usage of distinctiveness does not always distinguish contrast and assimilation (Moskowitz \& Skurnik, 1999). Second, the idea of individuating information has been widely used in the stereotyping literature and captures the idea that one may evaluate a person based on the individual's or the group's characteristics. Finally, we think that this term is more readily linked to within-category variance, and it is this perceived heterogeneity versus perceived homogeneity that may best predict whether contrast or assimilation processes are triggered. 
Alternatively, will individuating information lead to contrast effects resulting from a tendency to compare group members to group norms? Furthermore, guided by extant research, we hypothesized that when individuating information is poorly learned, participants will rely on learned categorical stereotypes to inform them about individual values, resulting in an assimilation effect. ${ }^{1}$

We used three approaches to examine the individuation hypothesis. First, we manipulated which dimensions were learned contingently or supplementally, with the assumption of heightened individuation for the contingently learned dimension. Second, in Experiments 2 and 3 we manipulated the relative salience of eye gaps and nose widths by reducing the range of variation on nose widths, with individuation likely to be greater for the more salient (wider range) dimension. Third, and related to the first two, we developed a way to measure individuation for each participant by the degree to which ratings differentiated group members along the relevant dimension. These individuation scores for each judgment task could then be used to test whether the manipulations of focal task and salience affected individuation. Moreover, they served as a way to test the individuation hypothesis by comparing context effects for high- and low-individuating participants. Our prediction was that low individuation would lead to assimilation and high individuation to contrast, which we tested using group differences as well as correlational analyses. Our manipulation of dimensional salience of eye and nose variations also allowed us to test whether illusory correlation effects might occur on the low-salient dimension (Chapman \& Chapman, 1969, 1971).

\section{EXPERIMENT 1}

Experiment 1 used materials and procedures modeled after Pettibone (2000). We constructed stereotypes based on members belonging to one of two fictitious groups, designated as gnomes and leprechauns. Each individual was associated with distinct cues, including group membership, a name, a specific facial configuration, and behavioral statements linked to likableness. Just as a person's gender and race are clearly apparent, group membership was obvious and unambiguous, as gnomes always ap- 
peared in red clothing and leprechauns always appeared in green clothing. Examples of gnomes and leprechauns are shown in Figure 2. The faces varied in two diagnostic ways: The width of the nose and the width of the gap between the eyes. Participants learned five faces from each of two groups (gnomes and leprechauns), with three target faces sharing the same critical features. Group stereotypes were developed from exposure to group members, as members of one group (e.g., gnomes) typically had narrower values for one facial feature, while members of the other group (e.g., leprechauns) had wider corresponding values.

During the course of learning, participants were exposed to supplemental information relevant to forming social evaluations of the group members. Specifically, three behavioral statements varying in valence (positive, neutral, or negative) were associated with each group member. To convey character rather than isolated instances, words such as "often," "usually," and "frequently" were included in the behavioral statements. For instance, one group member may have been described by the following three statements: "He frequently starts fights at school," "He goes to the bank every Monday morning," and "He repeatedly lies to his friends." The structure of the behavioral information was such that either the gnomes or leprechauns were generally more positive or more negative. This distribution of the behavioral information was designed to allow for the formation of group stereotypes. The particular structure is presented in the top section of Figure 3, with one group more likable than the other. We expected differences in how well this supplemental information was learned by each participant to be reflected in their individuation scores on this dimension.

In the induction phase, participants learned to associate names to specific facial configurations for each group and were presented with the supplemental behavioral information pertaining to likableness. In the judgment phase, names served as cues so that judgments were based on the participant's memory for the relevant information about the individual. We used this approach for prompting judgments for three reasons. First, real-world judgments are often based on remembered values. When one is asked to judge an acquaintance on a given dimension, the relevant information is rarely directly available but rather must be re- 

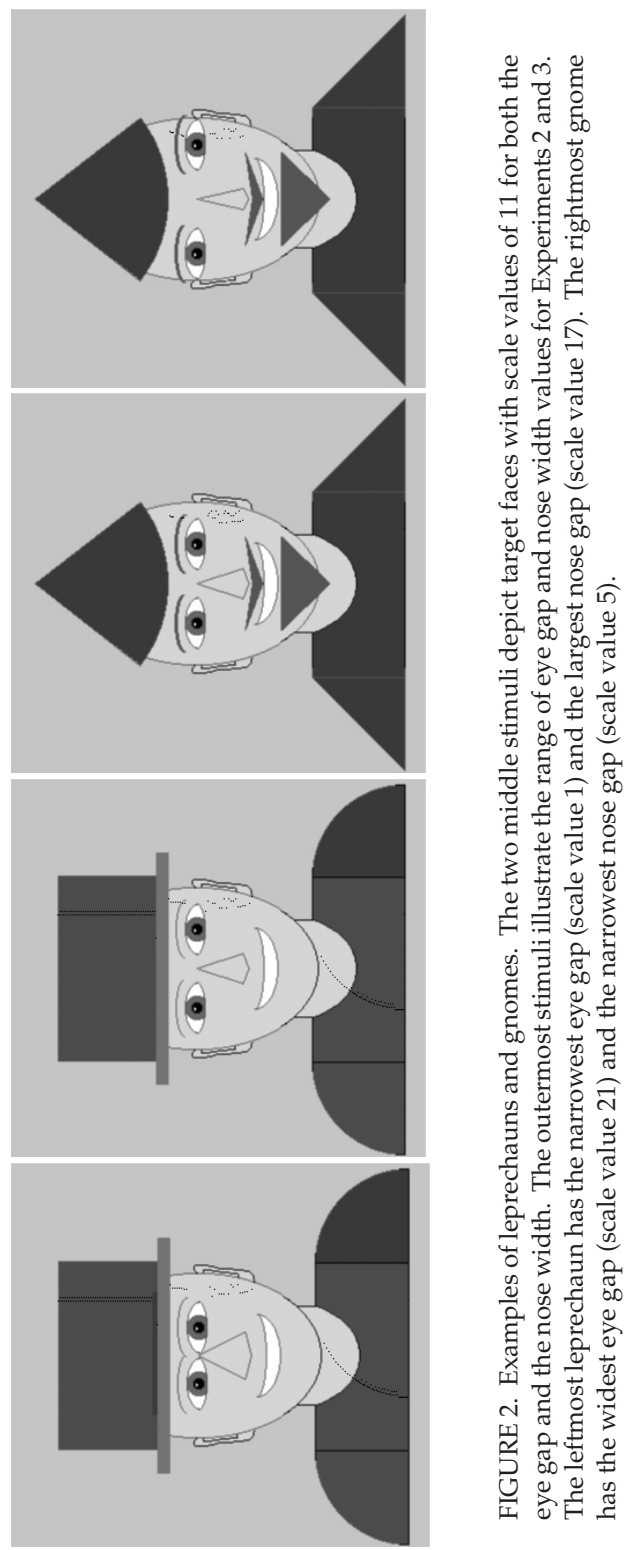


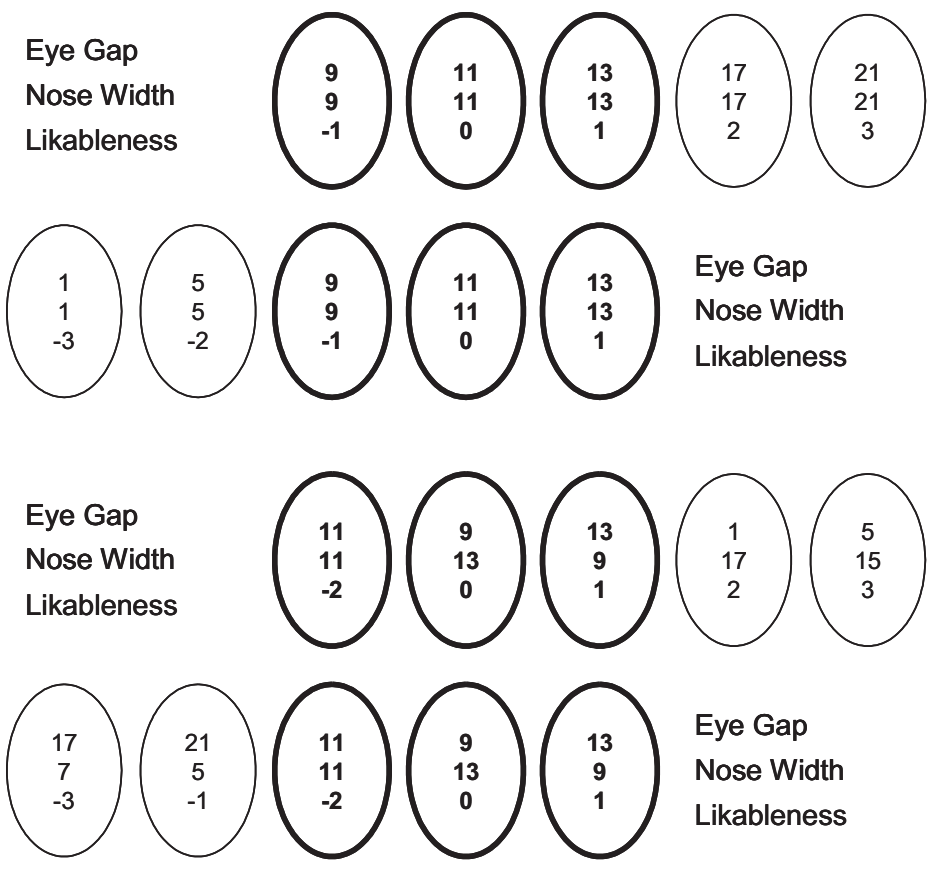

FIGURE 3. Each oval represents a unique category member who is characterized by a specific eye gap, nose width, and likableness value. The top two rows refer to stimuli used in Experiment 1, whereas the bottom two rows refer to stimuli used in Experiments 2 and 3. Target values appear in bold.

trieved from memory. Second, our primary interest was on how individuation of information in memory would prompt different stereotyping processes. We believed that using name cues would lead to greater variation in the individuation of that information and hence provide data relevant to testing our hypothesis. Third, Pettibone (2000) has shown that context effects are large with name cues.

In the judgment phase, participants made dominance judgments of feature width (eye gap and nose width) and likableness, along with ideal-point judgments of the pleasantness of the faces. The individuation hypothesis predicts that the obtained effect for the eye gap, nose width, and likableness judgments would depend on participants' ability to discriminate among the individuals within a group on each dimension. Those with high individuation scores for a given dimension should produce con- 
trast and those with low individuation scores should tend toward assimilation effects. One way that we hoped to manipulate individuation was through the learning procedure. Because learning group member names was contingent on the feature width information, we assumed that this information would be better individuated in memory than the supplementally learned likableness information. We tested this assumption by comparing individuation scores for these dimensions. Based on previous work on context effects on ideals (Pettibone, 2000; Pettibone \& Wedell, in press; Wedell \& Pettibone, 1999; Wedell et al., 2005), we predicted assimilation of ideal points for each group as reflected in pleasantness ratings.

\section{METHOD}

Participants and Design. Fifty-two psychology undergraduates from the University of South Carolina volunteered for this experiment in return for course credit. The basic design for Experiment 1 was based on work by Pettibone (2000). The design consisted of two within-subject variables, group (narrow and wide features) and target faces (scale values 9, 11, and 13), along with two between-subjects factors, group distribution (leprechauns assigned to narrow and gnomes to wide or vice versa), and judgment task order (likableness, width and pleasantness judgments, or the reverse order). The two distributions for likableness were based on favorability (high or low), where low favorability was linked to narrow features. Additional manipulations of the behavioral information during the learning trials include the randomization of the order in which the behavioral statements appeared on the computer screen and the counterbalancing of the target set of behaviors. The ordering of the stimuli was randomized during the judgment phase.

\section{STIMULI AND APPARATUS}

Desktop computers presented the stimuli and instructions, and collected responses. Stimuli were modeled closely after Pettibone (2000). Participants encountered schematic faces on a computer screen from two groups, leprechauns and gnomes. Examples of 
the stimuli appear in Figure 2. The faces shared the same oval-shaped head, eyes, eyebrows, ears, smile, and neck. The groups differed on several dimensions. Gnomes wore red shirts and red pointy hats. Their shoulders were angular and they had goatees and mustaches. Leprechauns wore green shirts and green top hats. They had rounded shoulders and no facial hair. Specific features of interest were the varying eye gap, nose width, and valence of behavioral information associated with each of the gnomes and leprechauns. There were five different faces in each group. The names of the leprechauns were Crafty, Floppy, Jumpy, Lucky, and Pappy. The gnomes were Al, Bob, Gus, Jim, and Ken. The faces differed in eye gap and nose width for both groups. Eye gaps and nose widths were perfectly correlated, and the scale values include $1,6,9,11,13,17$, and 21 , where a scale value of 1 equals four pixels for nose and two pixels for eye, with each size increment corresponding to a two-pixel increase.

Each group member was further described by three valenced behavioral statements. Behavioral descriptions in Experiments 1 through 3 were drawn from a pool of valenced behavioral statements generated from a norming study where behaviors were rated in terms of likableness from very unlikable (1) to very likable (9). Of the 60 rated behaviors, 48 served as possible behavioral statements for the experiments and were grouped into positive, neutral, and negative categories. Twenty behaviors received ratings from 7.27 to 8.35 , with a mean of 7.83 , and were designated as positive behaviors. Another 15 behavioral statements were rated from 5.27 to 6.08 , with a mean of 5.69 , and designated as neutral behaviors. While it may be expected that the neutral behaviors should center around 5.00, Eiser and van der Pligt (1984) note that raters are more apt to give more positive ratings, which they term the positivity effect. Finally, 13 behaviors received ratings ranging from 1.41 to 2.49 , with a mean of 1.85 , and were designated as negative behaviors. The particular behavioral statements chosen for Experiments 1through 3 are presented in Table 1. Behavioral statements were carefully selected for each stimulus to ensure that the target stimuli were described by behaviors that were matched with respect to likableness values. For example, three behaviors with a mean value of 6.67 were matched with another set of three behaviors with a mean value of 6.67. Al- 
TABLE 1.Behavioral Statements Selected for Experiments.

\begin{tabular}{ll} 
Mean & Statement \\
\hline 8.35 & Is very willing to help others out \\
8.24 & Comforts his friends when they are sad \\
8.16 & Listens to his friends' problems \\
8.16 & Helps out at the local homeless shelter \\
8.14 & Takes out the trash for an elderly lady \\
8.11 & Works well with other people \\
8.08 & Is always making his friends laugh \\
6.32 & Owns a cat and a dog \\
6.19 & Watches the sunset everyday \\
6.19 & Hikes in the mountains \\
6.08 & Works a nine to five job \\
6.03 & Goes to the movies on the weekend \\
5.92 & Plays the guitar every Saturday \\
5.84 & Favorite dinner is macaroni and cheese \\
5.84 & Is a photographer for a magazine \\
5.78 & Frequently goes to Mexican restaurants \\
5.76 & Reads the newspaper in the morning \\
5.68 & Balances his checkbook once a week \\
5.65 & Takes art lessons from a local artist \\
5.62 & Went to the store on Tuesday \\
5.54 & Goes out to eat at least twice a week \\
5.49 & Has Tuesday afternoon meetings \\
5.46 & Is majoring in art history \\
5.43 & Drinks sweet tea with every meal \\
5.27 & Goes to the bank every Monday morning \\
1.95 & Makes fun of people behind their back \\
1.78 & Habitually takes things without permission \\
1.73 & Frequently starts fights at school \\
1.65 & Vandalizes property \\
1.62 & Makes trouble wherever he is \\
1.59 & Repeatedly lies to his friends \\
1.46 & Gambles away his family's money \\
1.41 & Often publicly humiliates people \\
\hline &
\end{tabular}

Note. Behavioral information for Experiments 1-3 were selected from this list. Ratings were made on a 1 to 9 scale of likableness.

though the behavioral statements were matched, targets differed across groups. These were counterbalanced so that a given set appeared equally often in wide and narrow contexts. The likableness values described in Figure 3 ranged from -3 to 3 . These values indicate the sum of the valences making up each set. For example, -3 corresponds to 3 negative behaviors, -2 corresponds to two negative behaviors and one neutral behavior, etc. 


\section{PROCEDURE}

Participants were tested in groups of one to six in a large room with six computers facing the walls and spaced approximately one meter apart. After completing an informed consent form, participants were given brief verbal instructions. Detailed written instructions were presented on the computer screen for participants to read at their own pace.

Next, to familiarize the participants with the leprechauns and gnomes and to aid the learning process, participants previewed the names, faces, and behavioral information of each gnome and leprechaun for three seconds. After the preview, participants began the learning trials. For all learning trials, the face appeared in the middle of the screen. On either side of the screen were panels containing the possible names, leprechauns on the left and gnomes on the right. The names were arranged alphabetically with respect to the width of eye gaps, from top to bottom, with the narrowest at the top. All responses were made using the mouse.

Participants learned each group separately-that is, they learned the leprechaun group members followed by the gnome group members (or the reverse order). Separate blocks of learning were used because context effects are greatest under this condition (Pettibone, 2000; Pettibone \& Wedell, in press). On any given trial, participants were presented with a face and asked to select the name that corresponds with the face. After selecting an answer, feedback was provided concerning accuracy. To facilitate learning, if the incorrect name was chosen, a self-paced screen appeared with a reminder of the face and the correct name. Following the feedback, the three behavioral statements were presented along with the face and the name of the corresponding leprechaun or gnome for three seconds.

Participants could exit the learning phase after completing a minimum of 49 trials and achieving the learning criterion of correctly naming 19 out of the last 20 faces. If this learning criterion was not attained, participants completed the entire seven blocks (105 trials). Next, participants made a series of judgments about the leprechauns and gnomes on several dimensions, with names judgment cues. The judgment phase occurred in one of two orders described in the design section. All judgments were made on 
nine-point scales. The rating scale for judgments of the feature widths ranged from very narrow (1) to very wide (9). The rating scale for the perceived pleasantness of the facial configuration ranged from very unpleasant (1) to very pleasant (9). The rating scale for the likableness judgments ranged from very unlikable (1) to very likable (9). Participants judged each stimulus twice on every dimension. At completion of the judgment phase, participants were thanked for their participation and debriefed.

\section{RESULTS}

Ratings of Feature Width. Because the eye gap and nose width ratings followed the same pattern of results, the data were collapsed across feature width and analyzed. ${ }^{2}$ Mean width ratings for target and contextual faces are presented in the left panel of Figure 4. As shown, target ratings from the low group are higher than those from the high group, representing a contrast effect for these stereotypes. We conducted repeated-measures analysis of variance (ANOVA) on the width ratings of the three target faces, with group (low and high features), and target face (scale values 9,11 , and 13) as within-subject variables. ${ }^{3}$

Strong contrast effects on feature width ratings were supported by a significant main effect of group, $F(1,51)=82.92, p<.001$. A significant main effect of target indicated simply that participants were able to distinguish the feature widths among target faces, $F(2,102)=192.99, p<.001$. A Target $\times$ Group interaction was significant, $F(2,102)=11.96, p<.001$. As shown in the left panel of Figure 4, contrast effects were smallest for the middle target, consistent with the end face in each category being displaced away from the other group members.

Ratings of Pleasantness. The middle panel of Figure 4 shows the

2. For all experiments, criteria were established to eliminate participants based on learning accuracy and reliability; however, the pattern of results remained unchanged, so all participants were included in the analyses.

3. Analyses for all experiments included the between-subject variables of the two pairings of group distribution (leprechauns or gnomes as the narrow or wide distribution), and the two judgment orders (likableness, width and pleasantness judgments, or the reverse order). Judgment order interacted with likableness ratings only in Experiment 2. We do not refer to these factors in the reported analyses. 


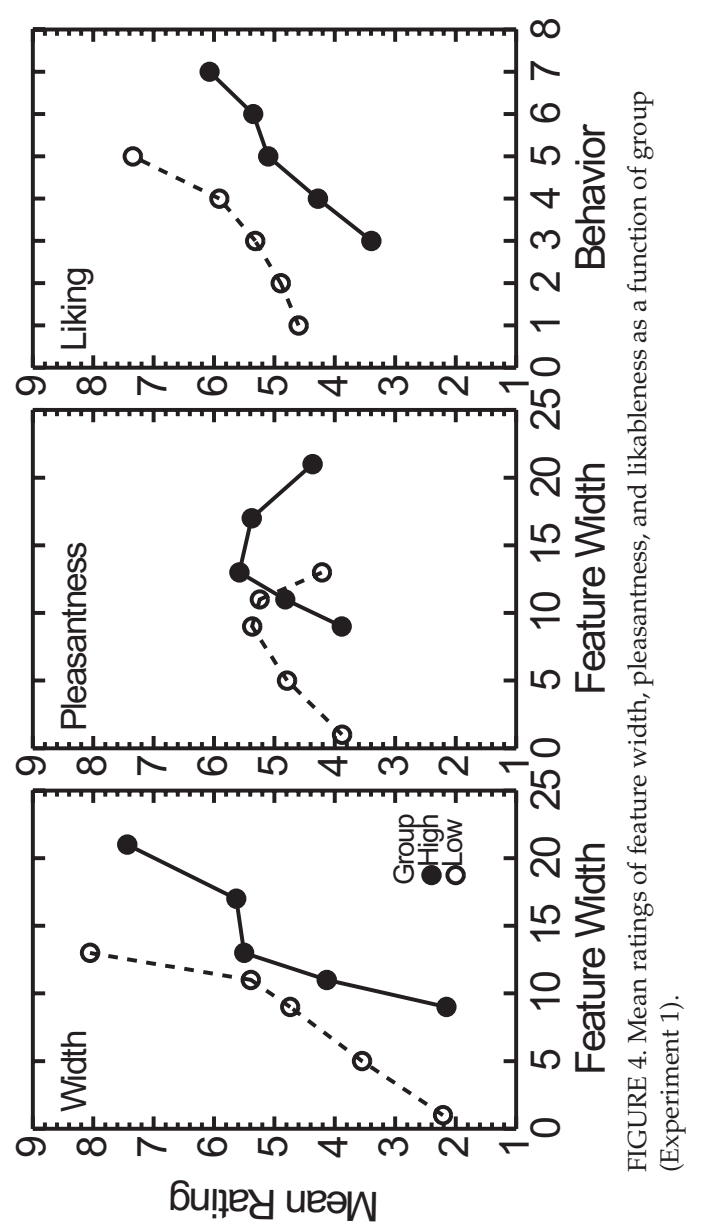


pleasantness ratings. In an initial set of analyses, we conducted separate ANOVAs for each context to provide statistical support for the ideal-point structure of pleasantness judgments. This structure is supported by the quadratic trend for faces in each context, which was highly significant for both low context, $F(1,51)=$ $16.18, p<.001$, and high context, $F(1,51)=12.15, p<.001$. Thus, both domains clearly demonstrate the anticipated single-peaked rating structure.

The crossover interaction shown in Figure 4 illustrates a disordinal reversal of preference, consistent with the assimilation of group ideals toward the average of the group members. A repeated-measures ANOVA was conducted on the pleasantness ratings of the three target faces. The Group $\times$ Target interaction was significant, $F(2,102)=5.82, p<.01$, reflecting assimilation of ideals. The linear component of the Group $\times$ Target interaction was significant, which supported the influence of an assimilative shift of the ideal-point, $F(1,51)=7.85, p<.001$.

Another way to evaluate the pleasantness ratings was to obtain an ideal point for each participant by inferring that the stimulus value rated the highest within the target range of 9 to 13 was the ideal point. A related samples t-test revealed that the ideal points for the two groups differed, $t(51)=3.41, p<.05, \mathrm{M}_{\mathrm{L}}=10.79$ and $\mathrm{M}_{\mathrm{H}}=11.87$.

Ratings of Likableness. The right panel of Figure 4 shows the likableness ratings as a function of behavior level and group. A repeated-measures ANOVA, parallel to those described previously, was conducted on the three target sets of behaviors. A main effect of target indicated that participants were able to differentiate the targets in terms of likableness, $F(2,102)=47.94, p<$ .001. More importantly, strong contrast effects were observed for likableness ratings, reflected in the significant main effect of group, $F(1,51)=36.94, p<.001$. Thus, the same target behaviors were judged more likable when exhibited by a member of the less likable group.

Individuation Scores and Context Scores. Individuation scores were designed to determine how distinctly members within each group were remembered. To calculate individuation scores, linear equations were developed. For convenience, the equations assumed equal spacing of stimuli on the rating scale. Linear 
individuation scores were computed using the expression, $-2 \mathrm{x}_{1}-$ $1 x_{2}+0 x_{3}+1 x_{4}+2 x_{5}$, where $x_{i}$ is the rating of the ith ranked stimulus in a contextual set. It was necessary to calculate a score for each group separately so that the score would not be confounded with context effects. Individuation scores were calculated for the two distributions (low and high groups) and then averaged to create an overall score as a measure of within-group individuation. A parallel procedure was used to calculate individuation scores for likableness ratings.

Because the behavioral information served as a supplemental dimension that was not contingently learned, we expected learning of the behavioral information to be poorer and hence individuation along this scale to be reduced. To test this assumption, we compared individuation scores for width ratings to those for likableness ratings using a related-samples $t$-test. This analysis showed that the contingently learned dimension of feature width was more individuated than the supplemental dimension of likableness, $t(51)=-6.24, p<.001$.

The key prediction of the individuation hypothesis is that contrast is more likely when individuation increases. To test this hypothesis, we examined the correlation between individuation scores and context scores, with context scores calculated by subtracting the mean of the target ratings in the low group from their mean in the high group. Thus, positive scores indicate assimilation and negative scores indicate contrast. A parallel procedure was used to calculate context scores for likableness ratings. In line with the individuation hypothesis, there was a significant negative correlation between individuation scores and context scores in the feature width domain, $r=-.61, p<.05$, and also in the likableness domain, $r=-.60, p<.05$. These negative correlations indicate that the greater the individuation among the group members, the greater the tendency toward contrast effects.

\section{DISCUSSION}

According to the individuation hypothesis, when group members are well distinguished in memory along the relevant dimension of judgment, contrast will likely occur, as their distinct values 
are compared to group norms. However, when members are not well distinguished on the relevant judgment dimension, group information will serve to disambiguate individuating information and assimilation will likely occur. The construct of individuation was both measured and manipulated in Experiment 1. Its measurement corresponded to how well ratings reflected differences in the attribute for group members. Its manipulation was based on the manipulation of focal versus supplemental information, with the focal dimension, which was important to the learning task, likely to lead to higher individuation than the supplemental dimension, which was irrelevant for learning. Differences in individuation scores verified this manipulation: Group members were better differentiated on the width dimension than on the likableness dimension.

For both the focal dimension of feature width and the nonfocal dimension of likableness, large stereotype-based contrast effects were observed. For width judgments, the same feature was rated wider when the target was a member of the low-width group. Similarly for likableness judgments, the same set of behaviors was judged more likable when the target was a member of the low-likableness group. The occurrence of contrast effects for both of these domains is inconsistent with the prevailing occurrence of assimilation to stereotype information found when applying preexisting stereotypes to new group members (Hilton \& von Hippel, 1996). We believe that this difference may be due to the greater information that participants in our study had about group members leading to relatively greater individuation and consequently a strong tendency to produce contrast. Although contrast prevailed, the tendency toward assimilation predicted by the individuation hypothesis still received some support, as context scores were significantly negatively correlated with individuation scores for both width and likableness. Thus, contrast was greatest for those who showed greatest individuation and reduced for those who did not distinguish among the group members well.

However, a problem for the individuation hypothesis was that despite the lower individuation for likableness scores, the overwhelming tendency was toward contrast. We would have predicted that a contrast should be greatly reduced in this condition, 
given the relatively low individuation. One possible explanation of this result lies in the simplistic nature of the design. The fact that three dimensions of variation (nose width, eye gap, likableness) were perfectly correlated may have led many participants to base their likableness judgments essentially on inferred behavioral values predicted by the perfect correlation with the other features. Even partial reliance upon such a strategy would yield the same contrast effects on likableness that occurred for feature width judgments. This problem is addressed by changes in the correlational structure among features used in Experiments 2 and 3. We anticipated that when participants could no longer rely on a simple correlational inference, assimilation would likely be observed when individuation was low.

In examining the results of Experiment 1, it is important to note that, as expected, the ideal-point domain of pleasantness of facial configuration demonstrated a qualitatively different type of stereotyping effect than found for the dominance judgment domains of width and likableness. Pleasantness ratings for the three targets showed a crossover interaction consistent with participants judging group members relative to a group ideal or stereotypic value. Individuals who shared the same defining facial features were evaluated very differently depending solely on their group membership. For the low-feature group, the ideal corresponded to a narrower feature value so that the widest target (13) was less preferred than the narrowest target (9). This preference relationship was reversed in the high-feature group. This shift in ideals replicates the finding reported by Pettibone (2000) with similar materials and procedures. More generally, it is consistent with contextual effects on ideal points produced when the recent context, rather than the categorical context, is manipulated (Wedell \& Pettibone, 1999; Wedell, et al. 2005).

The noted shifts in ideal points across groups may also be considered to parallel work on perceived attractiveness of faces (Langlois \& Raggman, 1990), in which the average of faces is perceived as more attractive than the constituent faces making up that average. As such, it appears that the ideal tends to drift toward the average of the group features. In fact, as Rhodes, Jeffery, Watson, Clifford, and Nakayama (2003) have suggested, group norms can quickly be readjusted or recalibrated. In their face ad- 
aptation paradigm, they ask participants to a rate a full set of faces in terms of attractiveness and normality of facial features. Then, after exposing participants to a subset of distorted faces, participants again rate the full set of faces. Results suggest that the preferred and most normal-looking face shifts toward the recently presented set of distorted faces. These various lines of research suggest that contextual norms or prototypes are likely to be viewed in a positive light so that individuals close to the norm receive heightened attractiveness.

Conversely, this result also suggests that individuals may be penalized to some degree for deviating from group norms or stereotypes. Target face 13 was further from the norm for the low group than for the high group and so was preferred less in the low than the high group. Consider groups who may differ in how they dress, such as lawyers and bikers. If members of these groups were rated in terms of how appropriately they were dressed, it is likely that a formally dressed biker would be viewed as less appropriately dressed than the casually dressed biker, but the reverse would be true for lawyers. Thus, group members may be judged relative to norms for their group, with deviation from the norm leading to a negative evaluation.

Finally, it is important to note that the affective reactions to individuals were driven by different processes in likableness judgments and pleasantness judgments (right and middle panels of Figure 3). For likableness judgments based on a dominance relationship (number of positive attributes), the stereotype was used as a standard of comparison against which group members were evaluated. Likableness associated with a given behavior was increased to the degree that the stereotypic behavior was less likable. However, targets retained their same ordering across contexts. On the other hand, pleasantness of faces increased as a function of the proximity to the stereotype, resulting in different orderings of target values across groups.

\section{EXPERIMENT 2}

A primary aim of Experiment 2 was to replicate Experiment 1 while disrupting the perfect positive correlation between feature widths and likableness of behaviors. This new design tests 
whether the contrast on likableness judgments was due to the artificial correlational structure of Experiment 1. A second aim was to examine the effects of manipulating feature salience.

The structure of the behavioral information was changed to reduce the correlations with other features under the constraint that one group would be perceived as more favorable. Thus, the correlation between likableness and eye gaps was changed to $r=-.68$ in Experiments 2 and 3 . If the disruption of the correlated structure leads participants to base their likableness judgments on remembered behaviors, then we anticipate some trend toward assimilation, consistent with the individuation hypothesis.

As shown in the bottom two rows of Figure 3, in Experiment 2 eye gap and nose were negatively correlated, and the range of nose widths was severely restricted in comparison to the range of eye gaps. The idea here was to distinguish whether participants are learning information pertaining to eye gap, nose width, or both. If participants only learn eye gap information (as it is now the more salient feature), they may show assimilation effects on nose width judgments, or they may assume a positive relationship and produce judgments consistent with this assumption, an illusory correlation effect.

In light of these changes, we expected greater individual differences in how the eye gap, nose width, and behavioral information would be learned and applied in judgment. As Wedell and Pettibone (1999) found assimilation of ideals when manipulating only the size of nose widths or only the size of eye gaps, we expect assimilation of ideals for pleasantness judgments, even if participants do not demonstrate proper learning of one of these dimensions.

\section{METHOD}

One hundred and fifty previously untested undergraduates from the University of South Carolina's psychology participant pool volunteered for this experiment in return for course credit. The method was nearly the same as Experiment 1, with a few noteworthy exceptions. The procedure for learning the category members by matching the name cue to the facial configuration re- 
mained the same. However, eye gap and nose width were negatively correlated. Furthermore, likableness was less correlated with either facial feature, $(r=.68$ and $r=-.68)$. The eye, nose, and behavioral values used are shown in the bottom two rows of Figure 3. As in Experiment 1, the behaviors associated with the gnomes and leprechauns were chosen from the same pool of valenced, behavioral statements. Again, in the judgment phase, participants were asked to judge the likableness, pleasantness of facial configuration, eye gap, and nose width for each category member.

\section{RESULTS}

Due to the increased complexity in the design of Experiment 2, we expected individual differences in how stereotypes would be used. Because eye gaps and nose widths were negatively correlated, the correlation of participants' eye gap ratings and nose width ratings provided one meaningful way to appropriately divide participants into feature-correlation groups. Surprisingly, although most correctly showed a negative correlation between the feature judgments, a large minority showed a positive correlation, consistent with an illusory correlation effect. We conservatively tested whether these positive correlations occurred more often than expected by chance by classifying participants into one of two groups based on the correlation between width ratings of nose and eye gap. Given the null hypothesis, $\rho=0.0$, the critical value for a one-tailed test at $\alpha=.05$ is $r=.549$. We classified participants by whether their correlation exceeded $r=.549$, with $5 \%$ or 8 participants expected to fall into this category. Instead 38 participants had correlations that exceeded the cutoff, which was significantly greater than chance, $\chi^{2}(1)=300.00, p<.001$. Based on this analysis, we elected to use cutoffs of $r=.549$ and $r=-.549$ to group participants into three feature-correlation groups: Positive, low, and negative. Note that despite many participants falsely perceiving a positive rather than negative correlation between features, most participants $(83 \%)$ were still able to meet the learning criteria as opposed to completing the entire learning block. Thus, 
many of these participants apparently learned to identify group members using a single feature.

Ratings of Eye Gap. The left panels of Figure 5 show the mean ratings of eye gap segregated by Feature-Correlation Group (Negative-Correlation Group, $N=52$; Low-Correlation Group, $N$ $=60$; Positive-Correlation Group, $N=38$ ). A repeated-measures ANOVA was conducted on the descriptive ratings of eye gap width, with the same within-subject variables as in Experiment 1, and with the addition of feature-correlation group as a between-subject variable. A main effect of group reflected strong contrast effects on width ratings of eye gap, $F(2,147)=167.83, p<$ .001. A significant Group $\times$ Feature-Correlation Group interaction indicated that the contrast effect was moderated by feature-correlation group, $F(2,147)=3.55, p<.05$. An interaction contrast demonstrated that this interaction may be attributed to a greater contrast for the negative- and positive-correlation group than for the low-correlation group, $F(1,147)=5.26, p<.05$. Additional significant effects included a main effect of target and a Target $\times$ Feature-Correlation Group interaction. However neither of these were particularly relevant to the hypotheses we tested.

Ratings of Nose Width. Mean nose ratings are provided in the middle panels of Figure 5. The same within-and between-subject variables were used when conducting a repeated-measures ANOVA on nose width ratings of the three target faces. A main effect of group reflected an overall contrast effect on ratings of nose width, $F(1,147)=7.88, p<.01$. However, group interacted with feature-correlation group, which suggested that the direction of these contrast effects depended on the feature-correlation group, $F(2,147)=55.81, p<.001$. Simple effects analyses indicated that the contrast effect was significant for the negative feature-correlation group, $F(1,51)=115.82, p<.001$. For the positive feature-correlation group, an apparent assimilation effect was significant, $F(1,37)=31.68, p<.001$. The group effect was not significant for the low feature-correlation group.

The very large Target $\times$ Feature-Correlation Group interaction, $F(4,294)=68.38, p<.001$, indicated that the groups distinguished among the target values differently. More specifically, simple effect analyses of the negative-correlation group indicated that mean ratings for the target stimuli ascend significantly, $F(2,102)=$ 

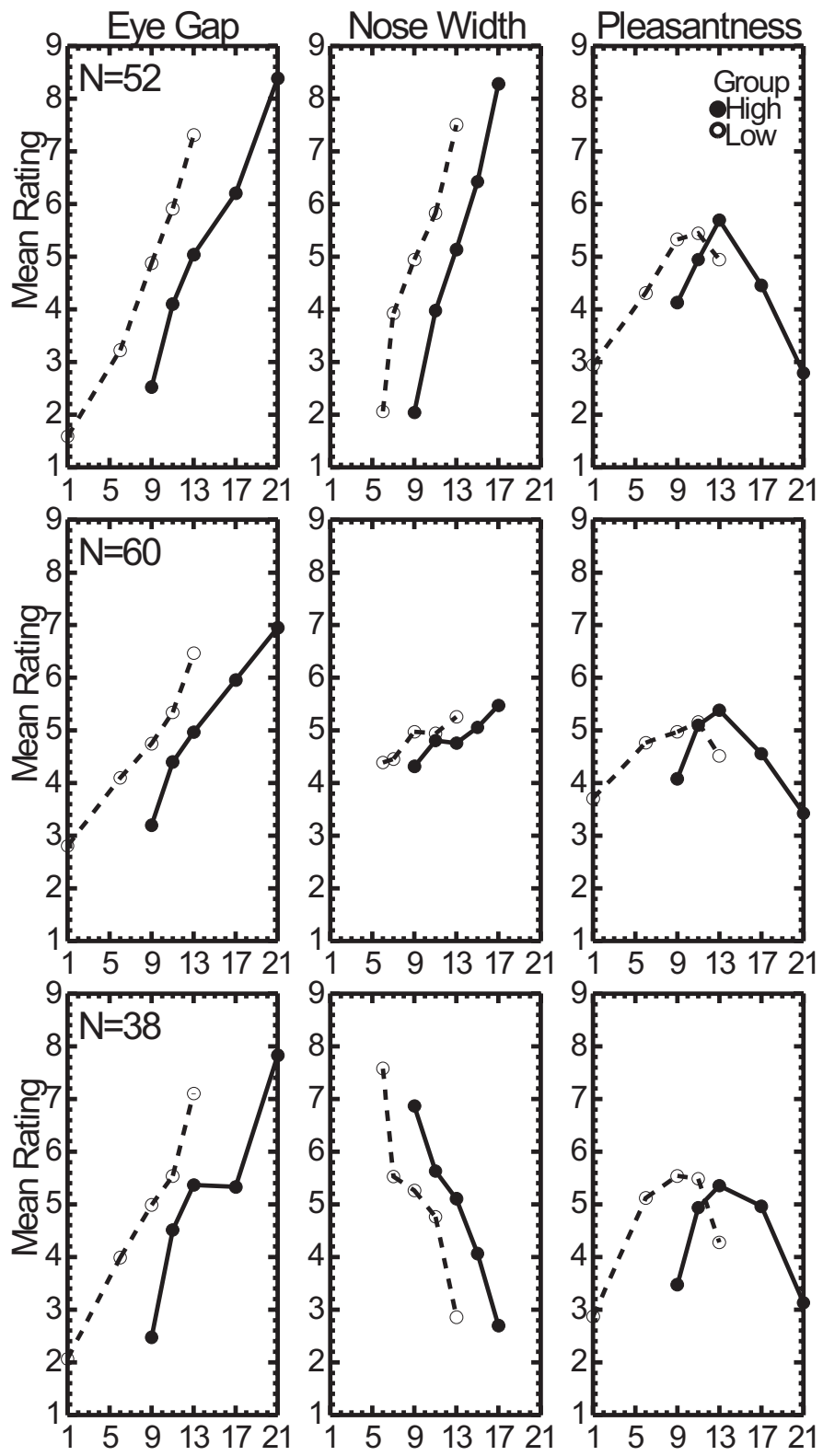

FIGURE 5. Mean ratings of eye gap, nose width, and pleasantness as a function of group (Experiment 2). The first, second, and third row of graphs represent the mean ratings of participants with a negative correlation, low correlation, and positive correlation of eye gap and nose width, respectively. Column headings indicate which dimension is being rated. 
$145.89, p<.001$. The target stimuli for the low-correlation group were undifferentiated, $F(2,118)=1.56, p>.05$. For the positive-correlation group, it was clear that the mean ratings of the target stimuli descend significantly, $F(2,74)=49.14, p<.001$. Finally, a significant three-way Group $\times$ Target $\times$ Feature-Correlation Group interaction was found, $F(4,294)=5.01, p<.001$, but this simply indicated some small difference in context effects for the targets across the grouping variable.

Ratings of Pleasantness. The mean ratings of the pleasantness of the facial configuration for the contextual and target stimuli are shown in the right panels of Figure 5. An initial set of separate ANOVAs for each context tested for the ideal-point structure of pleasantness judgments. This structure is supported by the quadratic trend for faces in each context, which was highly significant for both low context, $F(1,149)=78.00, p<.001$, and high context, $F(1,149)=115.33, p<.001$.

A repeated-measures ANOVA was then conducted on the pleasantness ratings of the three target stimuli. A main effect of target indicated that participants were able to distinguish the target stimuli, $F(2,294)=10.38, p<.001$. The crossover Group $\times$ Target interaction was consistent with a shift in the ideal point in the assimilative direction, $F(2,294)=40.07, p<.001$. A test of linear contrast reflecting a change in the ideal was significant, $F(1,147)=$ $54.88, p<.001$. These results generalized across feature-correlation groups. A related samples $t$-test conducted on inferred ideals indicated that the ideal point of the low context was significantly different from the ideal point of the high context, $t(150)=6.46, p<$ $.001, \mathrm{M}_{\mathrm{L}}=10.90, \mathrm{M}_{\mathrm{H}}=11.89$.

Ratings of Likableness. Due to a programming error in the likableness judgment phase, the stimuli were presented in an artificial, nonrandomized order for a subset of participants. The error was subsequently corrected so that analyses were conducted on the 50 participants who judged the stimuli in a randomized order. The same $2 \times 2$ repeated-measures ANOVA as used in Experiment 1 was conducted on likableness ratings. The only significant effect was that the main effect of target suggested that participants were capable of distinguishing the stimuli with respect to the valenced, behavioral information, $F(2,96)=24.75, p<.001$.

Individuation Scores and Context Scores. Individuation scores 
and context scores were calculated using the same procedure as used for dimensions in Experiment 1. As expected, eye gaps were better individuated than nose widths, as the eye dimension was the more salient dimension due to its extended range, $t(149)=$ $-8.40, p<.001$. The individuation hypothesis also predicts that the contingently learned dimensions will be more distinct in memory than supplementally presented dimensions. In support of this hypothesis, participants better individuated the group members in terms of their eye gaps than likableness, $t(149)=-13.15, p<.001$. However, the individuation of group scores for likableness and nose widths did not differ.

Correlations between individuation scores and context scores are reported for the three dominance-based domains. Supporting the individuation hypothesis, significant negative correlations between individuation scores and context scores of eye gap and nose width imply that stronger contrast effects were found for those who better discriminated the group members with regard to the specified dimension $(r=-.66, p<.05 ; r=-.82, p<.05)$. The correlation between context scores and within-group individuation of likableness was not significant, $r=-.23$, but it was in the direction predicted by the individuation hypothesis.

\section{DISCUSSION}

The imperfect correlation between feature widths and likableness was established in Experiment 2 to test whether contrast on the supplemental dimension of likableness was due simply to a perfect correlational structure. Clearly the change in the correlational structure produced a change in the likableness ratings. Whereas contrast was uniformly evident in Experiment 1 for likableness ratings, the results were nonsignificant in Experiment 2. This suggests that the contrast effect on the supplemental dimension of likableness in Experiment 1 was probably due to the artificially high correlation between widths and behavioral information. Participants appear to be highly sensitive to such correlations and use them in inferences about ratings.

Further evidence for the use of inferred correlational structure is found for the ratings of widths. Although eye gap and nose 
width were perfectly negatively correlated, participants varied strongly in whether they noted this correlation. Consequently, we divided participants into three groups based on the correlation between eye and nose widths judgments. The obtained contrast effects for the eye dimension were consistent with Experiment 1 and with past research (Pettibone, 2000). All three subject groups showed strong, robust contrast effects on eye gap judgments.

The nose judgment ratings were much less consistent. The results suggest that participants who paid attention and noticed the negative correlation of eye gap and nose width showed strong contrast effects on nose width judgments. Nonsignificant effects were noted when participants showed a low correlation between features. The remaining participants showed apparent assimilation effects on nose width judgments. We say apparent assimilation because we interpret this result as reflecting participants' assumption of a positive correlation of eye gap and nose width, an illusory correlation effect. There is a great deal of research supporting the phenomenon of illusory correlations, showing that participants often perceive relationships that either do not exist or that are not as strong as what actually exists (Chapman \& Chapman, 1969, 1971; Hamilton \& Gifford, 1976). As expected, participants demonstrated better learning for eye gap information as it was the more salient dimension of the two facial features. Here, many participants seemed to believe that a positive correlation of eye gap and nose width existed (even though the exact opposite was true) and based judgments on this false belief-that is, participants may have mimicked eye gap judgments when making nose width judgments. Thus, the apparent assimilation may simply be a substitution of remembered eye values for nose values.

Experiment 2 provided evidence of the robust nature of assimilation of ideals for the two group norms. As in Experiment 1, different standards were applied to group members based on different ideals formed for the two groups. As it was apparent that the majority of participants paid more attention to the eye dimension, pleasantness ratings may be based solely upon the eye dimension in this case. This interpretation further supported strong assimilation effects of ideals regardless of feature-correlation group. Although the eye dimension may have driven the 
pleasantness ratings in this experiment, it should be noted that Wedell and Pettibone (1999) independently manipulated eye and nose dimensions and found that participants included both in their judgments. Hence, the current illusory correlation finding is not simply due to problems in perceiving nose widths.

Supportive of the individuation hypothesis predictions, significant negative correlations between individuation scores and corresponding context scores were found for both width rating domains. The correlation was not significant, but in the correct direction for likableness judgments. Because no isolated group showed clear assimilation, the individuation hypothesis prediction of assimilation for poorly individuated group members was again not verified.

\section{EXPERIMENT 3}

Experiment 3 tested the limits of the generalizability of ideal-point shifts by making facial features the supplemental dimension. Thus, Experiment 3 was nearly identical to Experiment 2 except for one key change. Here, learning was contingent on behavioral information and the facial configuration served as supplemental information. This alteration provided a test to determine whether ideal points on the pleasantness dimension would shift in the assimilative direction when the facial information was not critical for learning, essentially exploring the generality and robustness of ideal-point shifts.

The individuation hypothesis predicts contrast effects on the focal dimension of likableness under the stipulation of adequate individuation of the stimuli. Without proper learning of the stimuli in terms of likableness, the individuation hypothesis predicts assimilation effects. The individuation hypothesis predicts assimilation for the supplemental dimension of eye width ratings, at least for those participants showing low individuation on this dimension.

\section{METHOD}

One hundred and sixty psychology undergraduates, previously untested, from the University of South Carolina volunteered for 
this experiment in return for course credit. Experiment 3 was identical to that of Experiment 2 aside from the manner in which participants learned about the gnomes and leprechauns. Participants were presented with three pieces of behavioral information that were centered on the screen. The order of the three behavioral statements was randomized. Situated on either side of the behavioral statements were panels containing the possible names, leprechauns on the left and gnomes on the right. The names were arranged in an identical manner as in the previous studies. After selecting a response and receiving feedback, participants viewed the faces, which provided the supplemental dimension. The judgment phase was the same as in Experiments 1 and 2.

\section{RESULTS}

Although it was useful to group participants in Experiment 2 in terms of the participants' correlation between eye gap ratings and nose width ratings, this grouping was not useful for Experiment 3. Instead there were noticeable and substantial individual differences in how well each dimension was learned. Therefore, analyses were conducted with distinct subpopulations defined by the individuation scores for each dimension.

Evaluative Ratings of Likableness. Experiments 2 and 3 were conducted during overlapping time periods and hence the programming error described earlier occurred here as well. Therefore, analyses were conducted on the likableness judgments from only the 50 participants who rated the likableness of individuals in random order (rather than serial order). Participants with an individuation score greater than 11.25 were grouped together as the high-individuation group. Participants with an individuation score less than or equal to 11.25 were grouped together as the low-individuation group. Mean likableness ratings are presented in Figure 6 as a function of individuation group (Low, $N=26$; High, $N=24$ ).

A repeated-measures ANOVA was conducted on the evaluative ratings of likableness for the three target stimuli with group as a within-subject variable and individuation group as a between-subject variable. Although there was no main effect of 

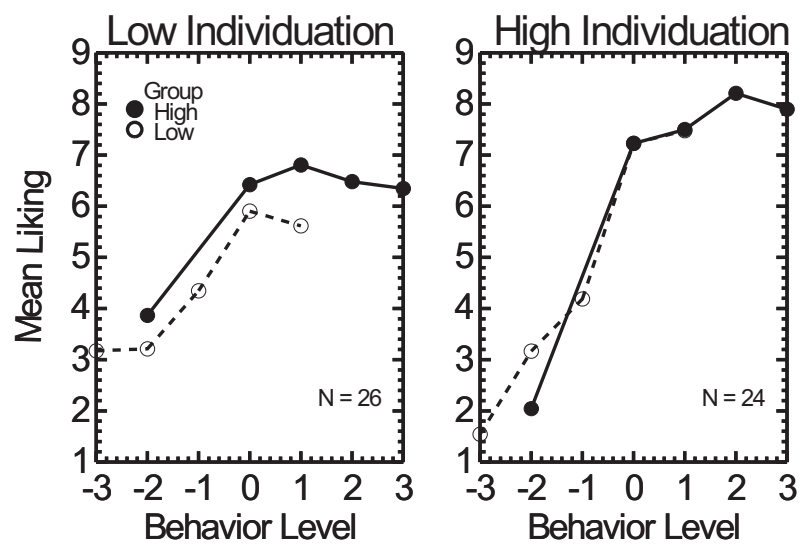

FIGURE 6. Mean ratings of likableness as a function of group (Experiment 3). The left and right graphs reflect low and high individuation groups, respectively.

group, there was a Group $\times$ Individuation Group interaction, $F(1$, $48)=7.16, p<.01$. Simple effects analyses indicate that the interaction is due to significant assimilation effects for the low-individuation Group $F(1,25)=5.85, p<.05$ and a nonsignificant trend toward contrast for the high-individuation group.

Eye Gap Ratings. Individuation scores on the eye gap dimension were used to group participants. The high-individuation group was comprised of participants with individuation scores greater than 4.25. The moderate group was comprised of participants with individuation scores greater than 0.00 and less than or equal to 4.25. The low-individuation group includes scores less than or equal to 0.00 . Figure 7 shows the mean width ratings of eye gap segregated by individuation group (Low, $N=61$; Moderate, $N=$ 44 , High, $N=55$ ). A parallel repeated-measures ANOVA was conducted on the descriptive ratings of eye gap width with individuation group as the between-subject variable.

Although there was not a main effect of group, there was a significant interaction of Group $\times$ Individuation Group, revealing that stereotyping effects depended upon how well participants 
discriminated group members' eye gap, $F(2,157)=14.55, p<.001$. As shown in Figure 7, contrast effects were observed for those who were able to best individuate eye gap, $F(1,54)=32.14, p<$ .001. A nonsignificant trend toward contrast was observed for the moderate-individuation group. Additionally, significant assimilation effects were observed for the low-individuation group, $F(1$, $60)=8.84, p<.01$.

Nose Width Ratings. Consistent with the nose width dimension being supplemental and of low salience, individuation scores were close to zero. Because this dimension was so poorly learned, we do not report an ANOVA on it.

Pleasantness Ratings. An initial set of separate ANOVAs for each context tested for the ideal-point structure of pleasantness judgments. Unlike Experiments 1 and 2, the quadratic trend analyses testing for this structure did not achieve significance, $F(1,159)=$ $3.77, p=.06$, for the low context and $F(1,159)=1.74, p=.19$, for the high context. These weak trends indicate the poor memory that most of the participants had for facial features.

A repeated-measures ANOVA was conducted on the pleasantness ratings of the three target faces with the same within-subject variables and the same between-subject variables as the analysis on eye gap. A significant main effect of target implies that participants were able to distinguish the target faces, $F(2,318)=17.78, p<$ .001. We note the absence of a Group $\times$ Target interaction, which suggests that there was not an assimilative shift of the ideal points. A t-test on ideal points also showed no significant effect of group.

Individuation Scores and Context Scores. Consistent with our assumption that individuation is better for contingently learned dimensions, individuation scores were higher for likableness than eye gap, $t(49)=-5.76, p<.001$, and nose width, $t(49)=-9.21, p<$ .001. Again, eye gap was better individuated than nose width because it was the more salient dimension, $t(159)=4.64, p<.001$.

Finally, providing further support for the individuation hypothesis, a significant negative correlation between context scores and individuation scores of likableness indicates greater contrast effects for those who better individuated the group members in terms of their likableness, $r=-.53, p<.05$. As in Exper- 

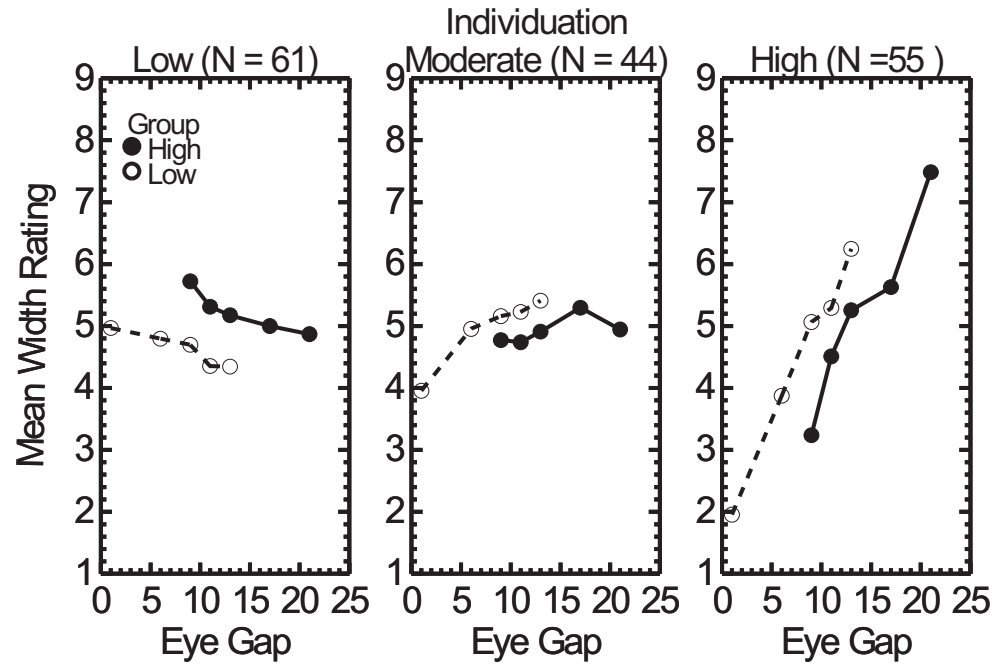

FIGURE 7. Mean ratings of eye gap as a function of group (Experiment 3). The left, middle, and right graphs reflect the low, moderate, and high individuation groups, respectively.

iment 2, significant negative correlations between context scores and individuation scores of eye gap and nose width were observed $(r=-.45, p<.05 ; r=-.32, p<.05)$.

\section{DISCUSSION}

Experiment 3 provided strong evidence in support of the individuation hypothesis. The individuation hypothesis predicts contrast effects when the stimuli are well individuated on a given dimension and assimilation effects when the stimuli are not well individuated. Overall, the p attern of results was in line with this prediction. As in Experiments 1 and 2, context scores were negatively correlated with individuation scores. However, unlike Experiments 1 and 2, clear evidence for assimilation was found for those participants who showed low individuation of group members. Significant assimilation was found for low-individuation participants for both judgments of likableness (Figure 6) and eye width (Figure 7). 
When comparing the results for eye gap ratings across Experiments 2 and 3, it is clear that the learning manipulation had important consequences. In Experiment 2, eye gaps served as a clear focal dimension so that participants generally showed strong individuation among group members and consequently contrast effects. By making eye gap a supplemental dimension in Experiment 3, it was consequently less well individuated. Consistent with the individuation hypothesis, the low-individuation group showed strong assimilation whereas the high-individuation group showed strong contrast.

Finally, Experiment 3 tested the generalizability of ideal-point shifts when the facial features were not learned contingently. Assimilation of the ideal points was not observed for the perceived pleasantness of the facial configuration. Although the low individuation of the eye and nose dimensions could contribute to the absence of the ideal-point shift, no shift was observed even for the high-individuation group. Therefore there is no clear evidence for spontaneously generating ideals for the supplemental dimension.

\section{GENERAL DISCUSSION}

These experiments were designed to test the proposition that how a stereotype is learned may be a critical factor in how the stereotype is applied in judgment. The current results have implications for the conditions under which stereotypes are used as comparison standards or as interpretative frames, yielding contrast or assimilation effects, respectively. Comparison of the results from Experiments 2 and 3 speaks directly to the effect of the learning environment, as these experiments differed only in the information that was contingently learned. These results generally support the individuation hypothesis, which states that high individuation among group members tends to lead to contrast and low individuation tends to lead to assimilation. When participants made distinctions among the group members, it appears that they used stereotypes as comparison standards, and hence they typically exhibited contrast effects. These contrast effects occurred even on supplemental dimensions such as the significant 
contrast effect on eye gap ratings in Experiment 3 for the high-individuation participants.

Regardless of whether a dimension was learned contingently or presented supplementally during the learning phase, assimilation effects were more likely when information was not well individuated. In Experiment 3 both the supplemental dimension of eye gap and the contingently learned dimension of behavioral likableness exhibited significant assimilation for low-individuation participants. Furthermore, in all three experiments there was a negative correlation between context scores and individuation scores, consistent with the individuation hypothesis. Comparison of results from Experiments 2 and 3 demonstrate how manipulating the focal and supplemental dimensions can affect individuation and thereby the nature of the context effects. When eye gap was a focal dimension (as in Experiments 1 and 2), individuation was high on this dimension and contrast was generally observed. However, when eye gap was a supplemental dimension (as in Experiment 3), individuation generally suffered and those who poorly individuated showed assimilation. The role of individuation is consistent with theories that point to the key role of individuating information in how stereotypes are used (Brewer, 1988; Brewer \& Feinstein, 1999; Fiske \& Pavelchak, 1986). It is also consistent with models of contrast and assimilation that point to a key role of distinctiveness in determining which of these effects occur (Stapel et al., 1998; Stapel \& Winkielman, 1998).

Note that while the learning environment may dictate to some degree how well individuated these dimensions are (through manipulation of what is contingently learned or through manipulations of salience), we observed very large individual differences suggesting that individuation is dependent on processing that is idiosyncratic to the individual. Future research may examine whether these individual differences are predicted by need for cognition, working memory capacity, or other motivationally related variables.

A second key finding of these experiments was that low individuation may lead to illusory correlation effects rather than assimilation effects. When the nose width dimension was made less salient in these experiments, a significant proportion 
of participants misremembered nose widths as being positively correlated with eye gap. Note that the demonstration of the illusory correlation implies that blocking occurred in the learning phase: The salient cue of eye gap captured most of the associative strength for a subset of participants so that nose width values were blocked from being learned. Blocking is sometimes characterized as inhibitory learning in which the learning of one dimension prevents the learning of other dimensions (Dickinson, 2001). Blocking in learning can be adaptive in that it allows individuals to concentrate on critical information and disregard irrelevant information (Dickinson, 1980). Through blocking, individuals may have learned that the gnome, Ken, had a very wide gap between his eyes, but they did not notice or ignored his very narrow nose. The occurrence of illusory correlation supports the stereotyping process whereby individuals use a theory of what groups are like to make judgments about individual members (Fiske, 2004). A problem arises when the hypothesized relationship is assumed rather than learned and hence produces gross misrepresentations of group members, as was observed for nose width ratings of many participants in Experiment 2.

It is useful to consider our results within the framework of the shifting standards model proposed by Biernat and her colleagues (Biernat \& Manis, 1994; Biernat et al., 2003; Biernat et al., 1991). This model focuses on how context effects differ depending on the type of response scale, objective or subjective. Objective scales retain an external, stable meaning. For example, height judgments are often made in terms of inches and feet, and weight judgments in terms of pounds or ounces. Biernat and her colleagues suggest that the semantic meaning of the endpoints on subjective scales (like the rating scales in the current experiments) change or shift to augment individuation among category members. Extant research has shown that individuals use categorical knowledge when making judgments on either scale; however, the manner in which the categorical information is used varies according to the response scale. Specifically, categorical information is often used as an interpretative frame when judgments are made on objective scales, resulting in assimilation. On the other hand, categorical information is often used as a comparison stan- 
dard when judgments are made on subjective scales, resulting in contrast. In light of this model and previous findings, the assimilative effects demonstrated on the likableness dimension and the eye gap dimension of Experiment 3 are important to highlight. These assimilative effects lend credence to our findings, suggesting that they are not an artifact of the response scale.

Experiments 1 and 2 extended and replicated the effects reported by Pettibone and Wedell (in press), including contrast effects on the dominance judgments of feature width and assimilation of ideals for affective judgments of pleasantness. The assimilative shift of ideals replicated regardless of whether individuals misinterpreted the relationship between eye gap and nose width (as in Experiment 2), indicating the robust nature of this effect. Experiment 3, however, placed a boundary condition on development of separate ideals for each group. To date, research has not demonstrated that assimilation of ideals for separate group arises when the relevant dimension is not key to learning. Further tests are needed to examine the generality of this finding. The stereotyping results from the current set of experiments imply that for some domains individuals may be penalized for deviating from the stereotypic norm. We believe that stereotyping effects on ideal-point domains deserve further analysis. Based on the work by Wedell et al. (2005), judgments of body images associated with different groups may well be a fruitful avenue of research in this regard.

In conclusion, these experiments provided evidence that learning strongly influences how stereotypes are subsequently used in judgment. When individuals do not learn the individuating information well, stereotypes serve an informative function and assimilation effects or illusory correlations effects may result. When individuals learned the information moderately well, stereotypes may serve as comparison standards so that contrast effects occur in dominance-based judgments. These effects of stereotype content are most easily studied when novel stereotypes are learned. We advocate for more studies in which the experimenter can control the content of stereotypes. This approach allows us to under- 
stand the stereotype development process and how learning factors influence mental representations of groups. Combined with work on preexisting stereotypes, these data provide a broader picture of the entire stereotyping process. A goal for future research would be to test whether the same types of processes that apply to these simplified stimuli (gnomes and leprechauns) may also be employed when using real-world stereotypes.

\section{REFERENCES}

Alexander, M. G., Brewer, M. B., \& Livingston, R. W. (2005). Putting stereotype content in context: Image theory and interethnic stereotypes. Personality and Social Psychology Bulletin, 31(6), 781-794.

Ashmore, R. D., \& Del Boca, F. K. (1981). Conceptual approaches to stereotypes and stereotyping. In D. L. Hamilton (Ed.), Cognitive processes in stereotyping and intergroup behavior (pp. 1-35). Hillsdale, NJ: Erlbaum.

Biernat, M., \& Crandall, C. S. (1996). Creating stereotypes and capturing their content. European Journal of Social Psychology, 26, 867-898.

Biernat, M., Kobrynowicz, D., \& Weber, D. L. (2003). Stereotypes and shifting standards: Some paradoxical effects of cognitive load. Journal of Applied Social Psychology, 33, 2060-2079.

Biernat, M., \& Manis, M. (1994). Shifting standards and stereotype-based judgments. Journal of Personality and Social Psychology, 66, 5-20.

Biernat, M., Manis, M., \& Nelson, T. E. (1991). Stereotypes and standards of judgment. Journal of Personality and Social Psychology, 60, 485-499.

Blair, I. V. (2002). The malleability of automatic stereotypes and prejudice. Personality and Social Psychology Review, 6(3), 242-261.

Brewer, M. (1988). A dual process model of impression formation. In T. K. Srull \& R. S. Wyer (Eds.), Advances in social cognition (Vol. 1). Hillsdale, NJ: Erlbaum.

Brewer, M.B., \& Feinstein, A. S. (1999). Dual processes in the cognitive representation of persons and social categories. In S. Chaiken, \& Y. Trope, Dual-process theories in social psychology (pp. 255-270). New York: Guilford Press.

Chapman, L. J., \& Chapman J. P. (1969). Genesis of popular but erroneous psychodiagnostic observations. Journal of Abnormal Psychology, 74, 272-280.

Chapman, L. J. \& Chapman J. P. (1971, November). Test results are what you think they are. Psychology Today, 18-22, 106-107.

Cooke, A. D. J., Janiszewski, C., Cunha, M., Jr., Nasco, S. A., \& de Wilde, E. (2004). 
Stimulus context and the formation of consumer ideals. Journal of Consumer Research, 31, 112-124.

Coombs, C. H. (1964). A theory of data. New York: Wiley.

Deaux, K. (1985). Sex and gender. Annual Review of Psychology, 36, 49-81.

Dickinson, A. (1980). Contemporary animal learning theory. Cambridge, UK: Cambridge University Press.

Dickinson, A. (2001). The 28th Bartlett Memorial Lecture. Causal learning: An associative analysis. The Quarterly Journal of Experimental Psychology, 54B(1), 3-25

Eagly, A. H., Wood, W., \& Diekman, A. B. (2000). Social role theory of sex differences and similarities: A current appraisal. In T. Eckes \& H. M. Trautner (Eds.), The developmental social psychology of gender (pp. 123-174). Mahwah, NJ: Lawrence Erlbaum.

Eiser, J. R., \& van der Plight, J. (1984). Accentuation theory, polarization, and judgment of attitude statements. In J. R. Eiser (Ed.), Attitudinal judgment (pp. 43-63). New York: Springer-Verlag.

Fiske, S. T. (1998). Stereotyping, prejudice, and individuation. In D.T. Gilbert, S. T. Fiske, \& G. Lindzey (Eds.), Handbook of social psychology (4th ed., Vol. 2, pp. 357-414). Boston: McGraw-Hill.

Fiske, S. T. (2004). Social beings: A core motives approach to social psychology. Hoboken, NJ: John Wiley \& Sons, Inc.

Fiske, S. T., Cuddy, A.J.C., Glick, P., \& Xu, J. (2002). A model of (often mixed) stereotype content: Competence and warmth respectively follow from perceived status and competition. Journal of Personality and Social Psychology, 84, 878-902.

Fiske, S.T., \& Pavelchak, M. A. (1986). Category-based versus piecemeal-based affective responses: Developments in schema-triggered affect. In R. M. Sorrentino \& E.T. Higgins (Eds.), Handbook of motivation and cognition: Foundations of social behavior. New York: Guilford Press.

Hamilton, D. L., \& Gifford, R. K. (1976). Illusory correlation in interpersonal perception: A cognitive basis of stereotypic judgments. Journal of Experimental Social Psychology, 12, 392-407.

Hamilton, D. L., \& Sherman, J. W. (1994). Stereotypes. Handbook of social cognition (pp. 1-68).

Hamilton, D. L. \& Sherman, J. W. (1994). Stereotypes. In R.S. Wyer, \& T.K. Srull, (Eds.), Handbook of Social Cognition, (Vol. 1). Hillsdale, NJ: Erlbaum.

Hilton, J. L., \& von Hippel, W. (1996). Stereotypes. Annual Review of Psychology, 47, 237-271.

Holbrook, M. B., \& Anand, P. (1990). Effects of tempo and situational arousal on the listener's perceptual and affective responses to music. Psychology of Music, 18, 150-162.

Jackson, L. A., Hodge, C. N., Gerard, D. A., Ingram, J. M., Ervin, K. S., \& 
Sheppard, L. A. (1996). Cognition, affect, and behavior in the prediction of group attitudes. Personality and Social Psychology Bulletin, 22, 306-316.

Johannesen-Schmidt, M. C., \& Eagly, A. H. (2002). Diminishing returns: The effects of income on the content stereotypes of wage earners. Personality and Social Psychology Bulletin, 28(11), 1538-1545.

Kunda, Z., \& Sherman-Williams, B. (1993). Stereotypes and the construal of individuating information. Personality and Social Psychology Bulletin, 19,90-99.

Kwong See, S. T., \& Heller, R. B. (2004). Judging older targets' discourse: How Do age stereotypes influence evaluations? Experimental Aging Research, 30(1), 63-73.

Lippman, W. (1922). Public opinion. New York: Free Press.

Langolis, K.H., \& Roggman, L.A. (1990). Attractive faces are only average. Psychological Science, 1, 115-121.

Lott, B., \& Saxon, S. (2002). The influence of ethnicity, social class, and context on judgments about U.S. women. The Journal of Social Psychology, 142(4), 481-499.

Manis, M., Nelson, T. E., \& Shedler, J. (1988). Stereotypes and social judgment: Extremity, assimilation, and contrast. Journal of Personality and Social Psychology, 55(1), 28-36.

Manis, M., Paskewitz, J., \& Cotler, S. (1986). Stereotypes and social judgment. Journal of Personality and Social Psychology, 50(3), 461-473.

Moskowitz , G. B., \& Skurnik, I. W. (1999). Contrast effects as determined by the type of prime: Trait versus exemplar primes initiate processing strategies that differ in how accessible constructs are used. Journal of Personality and Social Psychology, 76(6), 911-927.

Pettibone, J. (2000). Multiple pathways for contextual recruitment in social judgment. (Doctoral dissertation, University of South Carolina).

Pettibone, J. C., \& Wedell, D. W. (in press). Of gnomes and leprechauns: The recruitment of recent and categorical contexts in social judgment. Acta Psychologica Scandinavica.

Rhodes, G., Jeffery, L., Watson, T. L., Clifford, C. W. G., \& Nakayama, K. (2003). Fitting the mind to the world: Face adaptation and attractiveness aftereffects. Psychological Science, 14, 558-566.

Riskey, D. R., Parducci, A., \& Beauchamp, G. K. (1979). Effects of context in judgments of sweetness and pleasantness. Perception $\mathcal{E}$ Psychophysics, 26, 171-176.

Stapel, D. A., \& Koomen, W. (1998). When stereotype activation results in (counter) stereotypical judgments: Priming stereotype-relevant traits and exemplars. Journal of Experimental Social Psychology, 34, 136-163.

Stapel, D. A., Koomen, W., \& Velthuijsen, A. S. (1998). Assimilation or contrast? Comparison relevance, distinctness, and the impact of accessible information on consumer judgments. Journal of Consumer Psychology, 7, 1-24. 
Stapel, D. A., \& Winkielman, P. (1998). Assimilation and contrast as a function of context-target similarity, distinctness, and dimensional relevance. Personality and Social Psychology Bulletin, 24, 634-646.

Wedell, D. H., Hicklin, S. K., \& Smarandescu, L. O. (2007). Contrasting models of assimilation and contrast. In D. Stapel and J. Suls (Eds.), Assimilation and contrast in social psychology. New York: Psychology Press.

Wedell, D. H., \& Pettibone, J. C. (1999). Preference and the contextual basis of ideals in judgment and choice. Journal of Experimental Psychology: General, 128, 356-361.

Wedell, D. H., Santoyo, E. M., \& Pettibone, J. C. (2005). The thick and the thin of it: Contextual effects in body perception. Basic and Applied Social Psychology, 27(3), 213-227.

Wittenbrink, B., Gist, P. L., \& Hilton, J. L. (1997). Structural properties of stereotypic knowledge and their influences on the construal of social situations. Journal of Personality and Social Psychology, 72, 526-543. 